\title{
Notfallleistungen der Krankhäuser belasten Honorare
}

- Nach einem Urteil des Bundessozialgerichts (BSG) am 6. Februar 2013 müssen die Kassenärztlichen Vereinigungen (KVen) die Notfallbehandlungen in Krankenhäusern schon jetzt nachvergüten, obwohl derzeit noch ein Gerichtsverfahren gegen den Beschluss des Bewertungsausschusses (BA) zur Höhervergütung dieser Leistungen anhängig ist (BSG, Urteil vom 6. Februar 2013, AZ: B 6 KA 50/12 B).

Bereits im Dezember 2009 war der BA einer Forderung des BSG nachgekommen, das Vergütungsniveaus in Krankenhäusern bei Notfallleistungen anzuheben. Das Ge- richt hatte damals entschieden, dass die Bewertung der Notfallleistungen für Krankenhäuser vom 1. April 2005 bis 31. Dezember 2007 auf das Niveau der Vertragsärzte angehoben werden muss (Az: B 6 KA 46/07 R), zur Finanzierung der Nachvergütung aber die Krankenkassen Nachschüsse zur Gesamtvergütung leisten müssen. Diese Finanzierungsregelung wurde vom GKV-Spitzenverband mit Klage vor dem Landessozialgericht Berlin-Brandenburg angegriffen, über die bislang nicht entschieden worden ist.

\section{MMW Kommentar}

Das BSG hält die Höhervergütung der Krankenhäuser für möglich und zumutbar. Allein der Gesichtspunkt, dass der KV derzeit eine Refinanzierung ihres Aufwandes für die nachträgliche Erhöhung der Honorierung bei den Krankenkassen nicht möglich sei, rechtfertige keine andere Beurteilung. Die BSG-Richter haben mit diesem Urteil gewissermaßen verfügt, dass niedergelassene Ärztinnen und Ärzte Krankenhäusern aus ihrem Honorarvolumen eine Art zinslosen Kredit gewähren müssen.

\section{Erweiterte Überweisungsmöglichkeiten für die Osteodensitometrie}

- Nach einem Beschluss des Gemeinsamen Bundesausschusses (G-BA) wird die Indikation zur Osteodensitometrie demnächst deutlich erweitert. Künftig kann die
Knochendichtemessung bei allen Patienten bereits bei einem Verdacht auf eine Osteoporose durchgeführt werden, wenn eine medikamentöse Therapie geplant ist. Die
Indikationserweiterung betrifft auch die bisher stark eingeschränkte Möglichkeit der Überweisung von Patienten durch den Hausarzt an einen entsprechenden Facharzt.

\section{MMW Kommentar}

Die Knochendichtemessung wurde im Dezember 1999 durch Beschluss des Bundesausschusses der Ärzte und Krankenkassen als Kassenleistung mit erheblichen Auflagen versehen. Die Krankenkassen durften danach die Kosten für eine solche Untersuchung nur für Patienten übernehmen, die einen Knochenbruch ohne entsprechendes Trauma (z. B. Spontanfraktur) erlitten hatten und bei denen gleichzeitig aufgrund der Untersuchungsbefunde und der individuellen Krankheitsgeschichte ein begründeter Verdacht auf Osteoporose bestand. In dem o.g. Beschluss vom 21.2.2013 hat der Gemeinsame Bundesausschuss nunmehr beschlossen, die Richtlinie zu Untersuchungs- und Behandlungs-methoden der vertragsärztlichen Ver- sorgung (MVV-RL) zu ändern. Danach kann die Osteodensitometrie mittels DXA (DualEnergy-X-ray-Absorptiometrie) auch dann zu Lasten der Gesetzlichen Krankenversicherung (GKV) abgerechnet werden, wenn aufgrund konkreter klinischer und anamnestischer Befunde zum Zweck der Optimierung der Therapieentscheidung eine Absicht für eine spezifische medikamentöse Therapie einer Osteoporose besteht. Die Untersuchung kann zum Zweck der Optimierung der Therapieentscheidung frühestens nach fünf Jahren wiederholt werden. Eine frühere Untersuchung ist dann möglich, wenn diese aufgrund besonderer therapierelevanter klinischer und anamnestischer Befunde geboten ist. Keine Kassenleistung ist die Osteodesitometrie, wenn sie rein zur allgemeinen Vorbeugung osteoporosebedingter Knochenbrüche eingesetzt wird. Darüber hinaus kann eine Osteodensitometrie zu Lasten der Gesetzlichen Krankenversicherung dann nicht erbracht werden, wenn diese zur Kontrolle während oder auch nach einer spezifischen Arzneimitteltherapie (Therapiemonitoring) erfolgen soll. Die Knochendichte ist nach Expertenmeinung zur Beurteilung des Therapieerfolgs diesbezüglich kein zuverlässiger Indikator, da eine Knochendichteveränderung unter Therapie nicht unbedingt auch eine Änderung des Frakturrisikos bedeutet. 